\title{
Development of Dimensional Relation of Surface Leakage Current in Correlation with Humidity and Applied Voltage Stress for a Contaminated Overhead Insulator
}

\author{
F S Abdullah",*, M A M Piah², N A Othman ${ }^{3}$, A Din ${ }^{4}$ \\ ${ }^{1}$ School of Electrical System Engineering, Universiti Malaysia Perlis (UniMAP), Malaysia \\ ${ }^{2}$ Institute of High Voltage and High Current, Universiti Teknologi Malaysia (UTM), Malaysia \\ ${ }^{3}$ Universiti Tun Hussein Onn Malaysia (UTHM), Malaysia \\ ${ }^{4}$ Universiti Teknikal Malaysia Melaka, Malaysia
}

Received September 5, 2019; Revised December 18, 2019; Accepted December 24, 2019

Copyright@2019 by authors, all rights reserved. Authors agree that this article remains permanently open access under the terms of the Creative Commons Attribution License 4.0 International License

\begin{abstract}
Many empirical relations are proposed to estimate the surface leakage current (LC) through the experimental and simulation works. However, there are less exploration on developing the empirical equation that describe the surface LC in correlation with humidity and applied voltage stress. In this paper, an empirical relationship between the surface LC with humidity and applied voltage stress is proposed by using Dimensional Analysis (DA) technique. DA introduces a systematic approach based on Buckingham Theorem to present a complex relationship between the physical quantities into a dimensionless set of products. For validation purpose, the results obtained from the proposed mathematical model are compared to the practical data from the previous researchers. The validation shows that the analytical results are within the range of experimental results. By further analyzing the quantitative error analysis; mean absolute error (MAE) and root mean square error (RMSE), the proposed model is found to be able to be used as a predictive model to estimate the performance of surface LC at different relative humidity and applied voltage stress and contribute in reducing the testing expenses.
\end{abstract}

Keywords Leakage Current (LC), Dimensional Analysis (DA), Insulator, Mathematical Modeling, Contamination

\section{Introduction}

Insulators in overhead transmission line are considered to be one of the critical concerns to ensure the electrical integrity of the power transmission system. Due to outdoor applications, insulators cannot always retain its hydrophobicity. When subjected to the polluted environment, insulator tends to be contaminated by the inert materials from the pollution. In wet conditions, the soluble salts contained in the contaminants will be dissolved and produce a thin layer of high conductive electrolyte and provide a path for the surface leakage current (LC) to flow. With the increasing layer of contaminations, the LC will also increase. This will heat up the insulator's surface that will cause the dry band to be formed and encourage the small discharge on insulator's surface [1]. As the LC increases, the size of the dry band also will increase leading to the dry band arcing and insulator flashover. One way to examine the insulator performance under polluted environment is by LC monitoring [2]. LC monitoring is able to generate an alarm about the probability of a dangerous flashover risk level and recommends the necessary maintenance actions [1].

Other than the combination of contamination and moisture, surface LC is also influenced by the other environmental and electrical factors. Much of previous work highlighted the experimental results in studying the effect of humidity and applied voltage stress on the surface LC. Contaminated insulator under atmospheric condition like humidity shows some variation on the LC flows. The density and amplitude of LC increase when insulator surface becomes wet by the effect of humidity [3]-[5]. Under high humidity, strongly hygroscopic material on insulator surface absorb more moisture and increase of the solvability and ionization ability by several times result in 
stronger discharge and the larger LC[6]-[8]. Under different applied voltages, high relative humidity tends to increase the LC[3], [5], [9]. Increase in voltage potential will increase the electric field distribution[10]. Free electron possesses strong energy under the influence of electric field so that their movement will be accelerated to encourage the ionization process and lead to a high LC [11].

There are also many empirical equations presented to estimate the surface LC based on experimental works such as regression method [12], extreme value theory [13] and dimensional analysis (DA) [14]. Though there are many studies on empirical equations to estimate the surface LC, the exploration of the humidity and applied voltage stress is still inadequate. Therefore, this paper emphasizes on the empirical relation between the surface LC with humidity and applied voltage stress by using DA technique. The dimensional model derived in this research is based on Buckingham Theorem and obeys the homogenous condition. The proposed empirical relationship is then verified with the practical data collected from the previous researchers.

\section{Dimensional Analysis (DA) modelling}

DA introduces the basic concept of expressing the relationship between the dimensionless group based on Buckingham Theorem[15]. This method helps simplifications in investigating complex phenomena and is able to present the effect of a certain variable when the effects of other variables are known. Buckingham Theorem presents the desired relation connecting individual magnitudes as an algebraic expression related to the dimensionless products by examining the number of parameters and the number of dimensions involved in a physical relationship [16], [17]. Testing on prototype always requires lots of labour work that sometimes contributes to costly mistakes, requires quite a long time and there are cases when the variables are not available or inaccurately known [17], [18]. Despite of the disadvantages, the use of dimensional model to simplify the design and testing of a physical system is very effective to overcome this problem and convey the results obtained from the model to the prototype [17].

Dimensional model is a device which is so relevant to the physical system[17]. The dimensional model is able to present the dominant parameters associated to the physical activity and recommends the appropriate correlation of the test parameter values to be used when executing the experiment. The observation on the dimensional model may be used to estimate the physical system potential in the desired respect accurately[19]. The dimensional model of a physical system developed by DA technique is able to be projected to the other physical variables that are dimensionally similar. Their behaviour also can be closely related and the results of measurements on either one can be projected to the other [17].

\section{Derivation of Dimensional Model}

Based on the literature, the surface LC flows on contaminated insulator are dependent on the dominant parameters derived from the environmental and electrical stresses such as humidity, electrolyte conductivity, applied voltage, environmental pressure, and insulator creepage distance. Table 1 shows the base dimension of the target parameters. Throughout the derivation, four base dimensions of SI unit standard system which are mass $(M)$, length $(L)$, time $(T)$, and charge $(Q)$ used. Among the dominant parameters listed in Table $1, I$ is the dependent parameter which represents the magnitude of the quantity of the independent parameters $\left(h, \sigma_{e}, V, p\right.$, $L)[16]$.

Table 1. Target parameters and base dimension

\begin{tabular}{cll}
\hline Parameters & Symbol & \multicolumn{1}{c}{ Base Dimension } \\
\hline Leakage Current (LC) & $I$ & $Q T^{-1}$ \\
\hline Humidity & $h$ & $M L^{-3}$ \\
\hline Electrolyte Conductivity & $\sigma_{e}$ & $M^{-1} L^{-3} T Q^{2}$ \\
\hline Applied Voltage & $V$ & $M L^{2} T^{-2} Q$ \\
\hline Environmental Pressure & $p$ & $M L^{-1} T^{-2}$ \\
\hline Insulator Creepage Distance & $L$ & $M^{-1} L^{-1} T^{2} Q$ \\
\hline
\end{tabular}

The dependent and independent parameters can be written in a relationship form as in equation (1) where $f$ is an unknown function.

$$
I=f\left(h, \sigma_{e}, V, p, L\right)
$$

All the above dominant parameters are arranged in a dimensional matrix in term of its base dimensions;

\begin{tabular}{c|cccccc} 
& $I$ & $h$ & $V$ & $\sigma_{e}$ & $p$ & $L$ \\
\hline$M$ & 0 & 1 & 1 & -1 & 1 & -1 \\
$L$ & 0 & -3 & 2 & -3 & -1 & -1 \\
$T$ & -1 & 0 & -2 & 1 & -2 & 2 \\
$Q$ & 1 & 0 & 1 & 2 & 0 & 1
\end{tabular}

The rank of the dimensional matrix is found to be 4 with the numbers of dominant parameters, $N_{P}=6$ and the numbers of base dimensions, $N_{d}=4$. By applying Pi Buckingham Theorem[17], the number of independent set of products ( $\Pi$ 's) is 2 . The dimensionless product can be formulated as in equation (2).

$$
\Pi=I^{\epsilon_{1}} \cdot h^{\epsilon_{2}} \cdot V^{\epsilon_{3}} \cdot \sigma_{e}^{\epsilon_{4}} \cdot p^{\epsilon_{5}} \cdot L^{\epsilon_{6}}
$$

where $\epsilon_{1} \ldots \ldots \epsilon_{6}$ are the exponent of corresponding parameters. By assigning $\sigma_{e}, V, p$ and $L$ as repetitive parameters, the two independent sets of products can be written as in equation (3) and (4). 


$$
\begin{aligned}
\Pi_{1} & =\left[M^{-1} L^{-3} T Q^{2}\right]^{k_{1}}\left[M L^{2} T^{-2} Q\right]^{k_{2}}\left[M L^{-1} T^{-2}\right]^{k_{3}}\left[M^{-1} L^{-1} T^{2} Q\right]^{k_{4} I} \\
\Pi_{2} & =\left[M^{-1} L^{-3} T Q^{2}\right]^{k_{1}}\left[M L^{2} T^{-2} Q\right]^{k_{2}}\left[M L^{-1} T^{-2}\right]^{k_{3}}\left[M^{-1} L^{-1} T^{2} Q\right]^{k_{4}} h
\end{aligned}
$$

Since the surface LC is considered as the dependent parameter, so;

$$
\left[T^{-1} Q\right]=\left[M^{0} L^{0} T^{0} Q^{0}\right]
$$

The power indices $k_{1}, k_{2}, k_{3}$ and $k_{4}$ in equation (3) and (4) are determined by equating the power of base dimension in (5) and solve the simultaneous linear algebraic equations. The resulting dimensionless products of $\Pi_{1}$ and $\Pi_{2}$ are presented in a dimensional set as shown below.

\begin{tabular}{c|cccccc} 
& $I$ & $h$ & $V$ & $\sigma_{e}$ & $p$ & $L$ \\
\hline$M$ & 0 & 1 & 1 & -1 & 1 & -1 \\
$L$ & 0 & -3 & 2 & -3 & -1 & -1 \\
$T$ & -1 & 0 & -2 & 1 & -2 & 2 \\
$Q$ & 1 & 0 & 1 & 2 & 0 & 1 \\
$\Pi_{1}$ & 1 & 0 & $-2 / 5$ & -1 & $4 / 5$ & $7 / 5$ \\
$\Pi_{2}$ & 0 & 0 & $2 / 5$ & 2 & $-19 / 5$ & $-22 / 5$
\end{tabular}

Finally, the obtained power indices are substituted and the complete equation of dimensionless products can be written as in equation (6) and (7).

$$
\begin{gathered}
\Pi_{1}=\frac{I}{\sigma_{e}}\left(\frac{p^{4} L^{7}}{V^{2}}\right)^{\frac{1}{5}} \\
\Pi_{2}=h \sigma_{e}^{2}\left(\frac{V^{2}}{p^{19} L^{22}}\right)^{\frac{1}{5}}
\end{gathered}
$$

Based on Buckingham Theorem, the resulting dimensionless product can be expressed as (8).

$$
\Pi_{1}=f\left(\Pi_{2}\right)
$$

Equation (8) is rearranged to present the relationship of surface LC to the other dominant parameters.

$$
I=\sigma_{e}\left(\frac{V^{2}}{p^{4} L^{7}}\right)^{\frac{1}{5}} \cdot f\left(\Pi_{2}\right)
$$

Therefore, the resulting dimensional and mathematical model of surface LC can be written as a function of environmental and electrical stresses as in equation (10) where $D_{k}$ is the corrective constant.

$$
I=D_{k}\left(\sigma_{e}{ }^{3} h\right)\left(\frac{V^{4}}{p^{23} L^{29}}\right)^{\frac{1}{5}}
$$

Since the main objective of this paper is to highlight the relationship between the surface LC with humidity and applied voltage stress, equation (10) is modified by treating the parameters $\sigma_{e}, p$ and $L$ as a constant. Finally, the relationship between the surface LC with humidity and applied voltage stresses can be further expressed as in equation (11).

$$
I=D_{k} h V^{\frac{4}{5}}
$$

From (11), it can be observed that the derived dimensional equation is dimensionally homogenous. The principle of dimensional homogeneity is crucial to test the consistency and the completeness of an equation in physical algebra [16]. Therefore, equation (11) is proposed to be used as a predictive model in estimating surface LC in this work.

\section{Validation of the Proposed Dimensional Model}

The proposed dimensional model for predicting the surface LC is verified with the data obtained from previous experimental results. The practical data obtained from the other researcher's experiment is plotted using Mathcad software, and the curve of the proposed dimensional model is developed to fit the graph from the previous experimental result. Quantitative error analysis techniques; mean absolute error (MAE) and root mean square error (RMSE) are applied to evaluate the error between the actual data and predicted dimensional model of surface LC and affirm the effectiveness of the proposed model.

Dimensional model validation at different relative humidity

From the proposed model in (11), all the dominant parameters are considered as a constant and added $I_{L o}$ to adapt the dimensional model to the experimental result whereas the relative humidity is varied. Therefore, the dimensional model can be further simplified as in equation (12), where $C_{1}$ is the corrective constant.

$$
I(h)=C_{1} \cdot h+I_{L o}
$$

The values of the surface LC at different relative humidity are calculated from equation (12) and fit it into the experimental data provided by Tousi et al. [2] by varying the corrective constant. Both results of dimensional model and previous experimental data are plotted together as shown in figure 1 . It can be seen that the dimensional model fitting is very close to the doted data from the experimental results with quite a low deviation. The model has a considerably low MAE of 0.0283 and RMSE of 0.0375 with reference to the experimental data. 


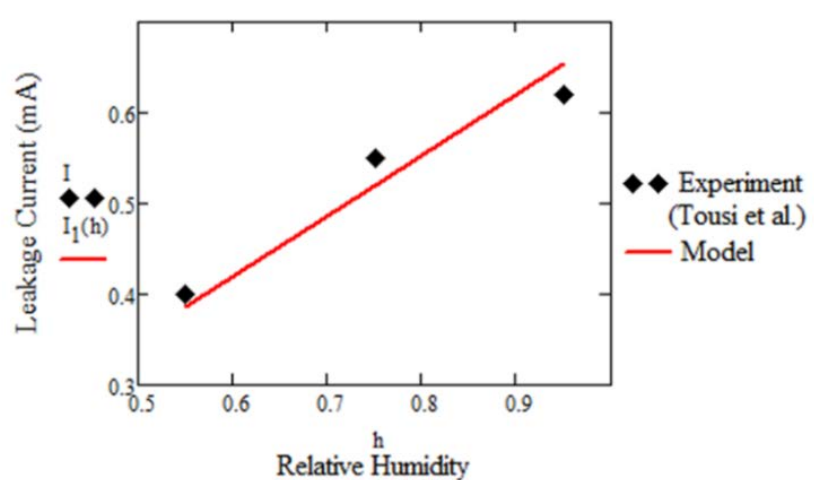

Figure 1. Variation of surface LC with the relative humidity

Dimensional model validation at different applied voltage

To validate the dimensional model at different applied voltages, equation (11) is simplified by assuming that the relative humidity is constant and written in a function of the applied voltage as in equation (13). From the simplified equation, it is found that the surface LC of the contaminated insulator is in linear relationship with the applied voltage.

$$
I(V)=C_{2} \cdot V^{\frac{4}{5}}+I_{L o}
$$

The proposed model is compared with the experimental data gathered from two previous researchers; Suwarno et al. [20] and Jin et al. [21]. Figure 2 shows the comparison of the surface LC from the dimensional model and previous experimental results. It can be clearly verified that the derived dimensional model is in a good agreement with the experimental results [20], [21]. From the quantitative error analysis (refer table 2), the error computed between the dimensional model and previous experiment is relatively low.

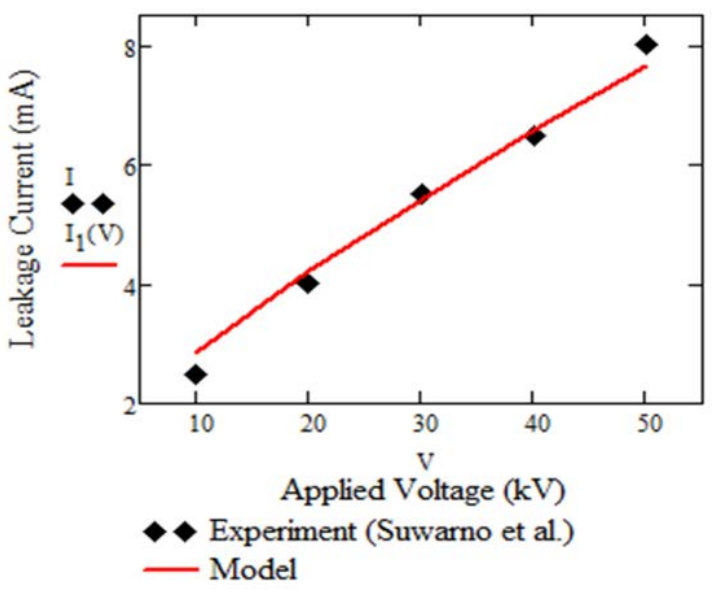

(a)

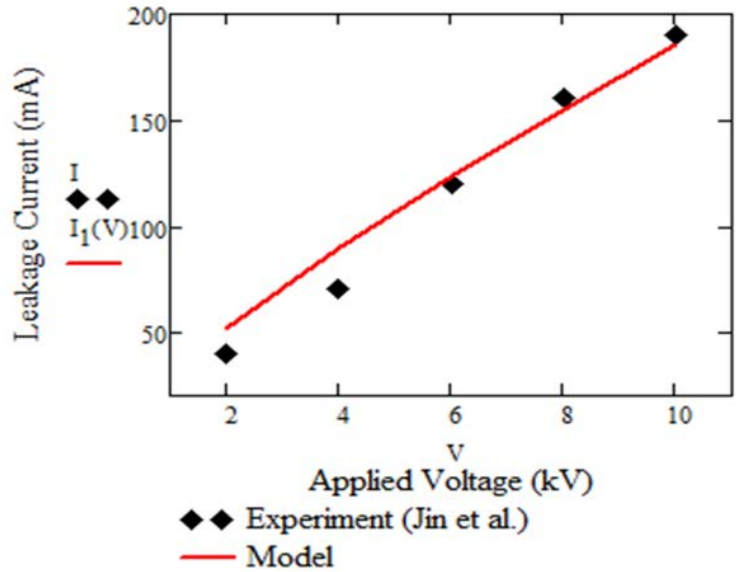

(b)

Figure 2. Comparison between dimensional model and experimental results at different applied voltage (a) Suwarno et al. (b) Jin et al.

Table 2. Quantitative error analysis

\begin{tabular}{ccc}
\hline Experiment & MAE & RMSE \\
\hline Suwarno et al. & 0.0517 & 0.0699 \\
\hline Jin et al. & 0.1277 & 0.1776 \\
\hline
\end{tabular}

Dimensional model validation at different relative humidity and applied voltage

To observe the effectiveness of the proposed model at different relative humidity and applied voltage, equation (11) is modified by adding $I_{L o}$ to adapt the dimensional model to the experimental results and verified with the previous experimental data by Jin et al. [21].

$$
I(h, V)=C_{3} h V^{\frac{4}{5}}+I_{L o}
$$

Figure 3 shows the variation of surface LC at different applied voltages for $50 \%$ and $98 \%$ relative humidity. Based on figure 3 , it is found that the proposed model is significantly in close agreement with the experimental results. In general way, it can be concluded that the relative humidity and applied voltage stress on contaminated insulators are capable of giving a significant effect on surface LC. The tendency of surface LC is in linear growth with the humidity and applied voltage. Under high relative humidity condition, the moisture absorption on insulator's surface increases which leads to high dissolution rate of the inert materials and leads to the formation of the conduction layer [22], [23]. Increasing the applied voltage will increase the electric field distribution and affect the LC variation [10]. The quantitative error analysis performed is presented in table 3. It is noticed that the MAE is substantially low which are 0.1001 and 0.1140 at relative humidity of $50 \%$ and $98 \%$ respectively. 


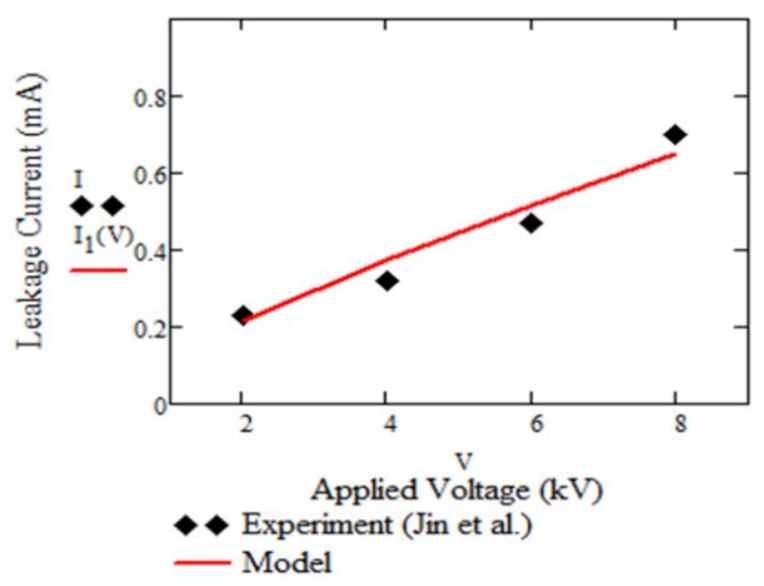

(a)

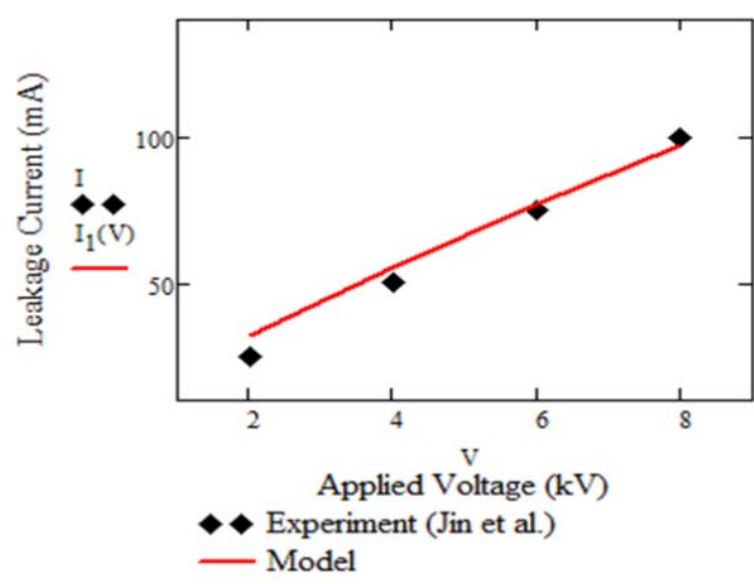

(b)

Figure 3. Verification of proposed dimensional model at different applied voltage and relative humidity (a) $50 \%$ (b) $98 \%$

Table 3. Quantitative error analysis for $50 \%$ and $98 \%$ of relative humidity

\begin{tabular}{ccc}
\hline Relative Humidity & MAE & RMSE \\
\hline $50 \%$ & 0.1001 & 0.1085 \\
\hline $98 \%$ & 0.1140 & 0.1543
\end{tabular}

\section{Conclusions}

This article demonstrates a mathematical approach based on DA technique to present the relationship between the surface LC of contaminated insulator with the humidity and applied voltage stress. DA model proposed in (11) can be considered to obey the dimensional homogeneity condition which makes it possible to test the completeness of an equation. The dimensional model shows a close agreement with the previous experimental results, thus ensuring that the model's ability to compute the surface LC in correlation with humidity and applied voltage stress. The effectiveness of the dimensional model is further affirmed by the quantitative error analysis. Throughout the analysis, the MAE shows a lower value compared to RMSE, which justifies the validity of the proposed dimensional model. The results also suggest that the dimensional model derived in this paper can be used as a predictive model that will assist in estimating the surface LC and contribute to the reduction of the testing expenses and labour work.

\section{Acknowledgements}

This research was supported by Research University Grant (UTM-TDR 46, Vot.Q.J130000.3551.07G60 and UTM-TDR 46.3, Vot.Q.J130000.3551.06G14) provided by Universiti Teknologi Malaysia. The first author would like to gratefully acknowledge School of Electrical System Engineering, Universiti Malaysia Perlis and the Ministry of Higher Education Malaysia (MOHE) for the financial assistance of this publication which makes this research viable and effective.

\section{REFERENCES}

[1] G. Montoya, I. Ramirez, and R. Hernandez, "The Leakage Current as a Diagnostic Tool for Outdoor Insulation,” 2008 IEEE/PES Transm. Distrib. Conf. Expo. Lat. Am., pp. 1-4, 2008.

[2] A. Azizi Tousi and M. Mirzaie, "The Effect of Severity and Location of Pollution on Leakage Current Characteristics of Porcelain Insulators under Different Humidity Condition,” J. Electr. Eng., vol. 13, no. 4, pp. 356-362, 2013.

[3] Suwarno and S. K. Ardianto, "Study on Leakage Current Characteristics of Epoxy Resin for Outdoor Insulators," Proc. 6th WSEAS Int. Conf. Power Syst. Port., pp. 201-206, 2006.

[4] Suwarno and S. K. Ardianto, "Leakage Current Waveforms and Arcing Characteristics of Epoxy Resin for Outdoor Insulators under Clean and Salt Fogs,” ITB J. Eng. Sci., vol. 40, no. 1, pp. 40-60, 2008.

[5] M. E. A. Slama and A. Beroual, "Behavior of AC High Voltage Polyamide Insulators: Evolution of Leakage Current in Different Surface Conditions," Adv. Electr. Electron. Eng., vol. 13, no. 2, pp. 74-80, 2015.

[6] C. Wang, T. Li, Q. Peng, Y. Tu, L. Zou, and S. Zhang, "Study of Composite Insulator Leakage Current Characteristics in Contamination and Humidity Conditions," 2014 IEEE Conf. Electr. Insul. Dielectr. Phenomena, CEIDP 2014, pp. 353-356, 2014.

[7] S. Y. Jing Lei, Xiangyang Peng, Chunyao Lin, Qiuwei Zheng, Siyi Liu, "Influence of Insoluble Substance Hygroscopicity on Insulator Leakage Current,” 2016 IEEE Int. Conf. High Volt. Eng. Appl., pp. 1-4, 2016.

[8] O. E. Gouda and G. M. Amer"), "Factors Affecting the Leakage Current Bursts of High Voltage,” 39th Int. Univ. Power Eng. Conf. 2004. UPEC 2004., no. 1, pp. 114-117, 2004. 
[9] Z. Jianbo, G. Bo, W. Qingliang, and Z. Qiaogen, "Leakage current pattern for diagnosing the contaminated degree of ceramic insulators under different humidity," Asia-Pacific Power Energy Eng. Conf. APPEEC, pp. 1-4, 2009.

[10] M. Dhofir, D. D. Andaru, and R. N. Hasanah, “The Influence of Conduction Band Location on the Leakage Current Level of a Glass Insulator,” 2017 5th Int. Conf. Electr. Electron. Inf. Eng., pp. 25-31, 2017.

[11] D. V. N. Diffni Gomez A M, Rafiq Mathersa AB, "Experimental and Simulation Studies on Room Temperature Vulcanization (RTV) Coatings By Inclined Plane Tracking and Erosion Test Method,” 3rd Int. Conf. Cond. Assess. Tech. Electr. Syst., pp. 302-305, 2017.

[12] M. A. Pinotti and L. H. Meyer, "Mathematical Model for Prediction of the Leakage Current on Distribution Insulators of $25 \mathrm{kV}$ class,” 2017 IEEE Electr. Insul. Conf., no. June, pp. 256-260, 2017.

[13] H. Ali, "Leakage Current Prediction for High Voltage Insulators Flashover based on Extreme Value Theory," 2016 Int. Symp. Comput. Consum. Control, pp. 870-873, 2016.

[14] M. A. M. Piah and A. Darus, "Modeling Leakage Current and Electric Field Behavior of Wet Contaminated Insulators,” IEEE Trans. Power Deliv., vol. 19, no. 1, pp. 432-433, 2004.

[15] Andrew C Palmer, Dimensional Analysis and Intelligent Experimentation, 1st editio. Singapore: Ltd, World Scientific Publishing Co. Pte., 2008.

[16] B.S. Massey, Units, Dimensional Analysis and Physical Similarity, 1st editio. London: Butler \& Tanner Ltd, 1971.

[17] T. Szirtes, Applied Dimensional Analysis and Modeling, Second Edi. United Kingdom: Butterworth-Heinemann, 2007.

[18] S. Sudalai Shunmugam, N. Vasudev, K. N. Ravi, and K. A. Venkatesh, "Applicability of Dimensional Analysis for the Prediction of Pollution Performance of Insulators: an Experimental Study,” IET Gener. Transm. Distrib., vol. 11, no. 5, pp. 1319-1324, 2017.

[19] A. S. Ahmad, S. Ahmed, S. S. Ahmed, and A. L. K. Oman, "Dimensional Analysis for Contamination Severity Assessment on High Voltage Insulators,” 2007 Int. Conf. Intell. Adv. Syst., pp. 893-896, 2007.

[20] Suwarno and S. Ardianto, "Characteristics of leakage current and flashover on epoxy resin insulator," Proc. 2014 IEEE Work. Electron. Comput. Appl. IWECA 2014, vol. 3, pp. 754-757, 2014.

[21] L. Jin, Z. Xu, and S. Zhang, "A pre-warning method of contamination flashover based on the leakage current of insulators in dry condition,” Proc. Int. Symp. Electr. Insul. Mater., vol. 2, pp. 757-760, 2017.

[22] N. A. Othman, M. A. Mohamed Piah, and Z. Adzis, "Space charge distribution and leakage current pulses for contaminated glass insulator strings in power transmission lines,” IET Gener. Transm. Distrib., vol. 11, no. 4, pp. 876882, 2017.

[23] Yuan tian and W. , Xiangtie-yuan, Zhangrui , Zhoujun, "Study on the Frequency Spectrum ofLeakage Current for the Pollution layer Dampness of Porcelain Post Insulator in the Pollution Test,” 2014 Int. Conf. Power Syst. Technol., pp. 1355-1362, 2014. 